\title{
Usage-based optimisation of characteristic maps for conceptual powertrain design
}

\author{
Tobias Hellberg $^{1}$ (1) $\cdot$ Martin Meywerk ${ }^{1}$
}

Received: 14 January 2016/Accepted: 3 April 2017/Published online: 17 April 2017

(C) The Author(s) 2017. This article is an open access publication

\begin{abstract}
With an increasing number of vehicles having alternative powertrains, the choice of the most appropriate powertrain for a vehicle class or load cycle is more challenging. This paper introduces a method for usage-based optimisation of powertrains. Based on a longitudinal dynamic simulation, the characteristic maps of the traction machines are optimised for different user cycles and these characteristic maps form the objective function of a minimisation problem. The goal of optimisation is to minimise well-to-wheel carbon dioxide emissions. Besides the conventional powertrain provided with petrol or diesel engine, battery electric, parallel-, serial- and power-split hybrid powertrains with assisting or dominating petrol and diesel engines are investigated. The results show that the proposed method delivers the optimal powertrain for a specific usage by applying an optimisation with reasonable restrictions.
\end{abstract}

Keywords Optimisation · Powertrain · Longitudinal dynamic vehicle simulation - Hybrid electric vehicle . Battery electric vehicle

\section{Introduction}

The powertrain configurations in passenger cars are characterised by an increasing diversity in recent years. The main reason is the intensified search for resource efficiency and the electrification of the powertrain. Internal

Tobias Hellberg

tobias.hellberg@hsu-hh.de

1 Helmut Schmidt University Hamburg, Institute of Automotive and Powertrain Engineering, Hamburg, Germany combustion engines (ICE) are still the standard propulsion system for vehicles, but there are numerous configurations with a combination of electric machines (EM) and ICE in a propulsion system: hybrid electric vehicle (HEV). Alternatively, the battery electric vehicle (BEV) uses an EM as sole traction machine. Consequently, the task to identify the optimal powertrain for a specific application has become more challenging. This paper proposes a method for an optimisation of powertrain components on usagebased load cycles. Therefore, powertrain-specific parameters are part of an optimisation problem that uses a longitudinal dynamic simulation to identify a parameter set which minimises carbon dioxide emissions for the considered load cycle.

To provide an optimal vehicle for a specific usage, an adaptation of vehicle components for the specific application is required. This leads to a usage-based dimensioning of powertrain components for hybrid, electric, and conventional powertrains.

Some approaches for dimensioning processes of hybrid components are published. In [18], the dimensioning methods are categorised into manoeuvre based and comparison of variants. The comparison of discrete variants with scaled powertrain components on different load cycles and their evaluation with regard to the objective is designated as comparison of variants. [12] chooses a discrete scaling for a Plug-In Hybrid on the urban dynamometer driving schedule (UDDS) with electrical range varying from 5 to 40 miles. Additionally, the power of the electrical machine is varied in a range of $\pm 40 \%$. The optimal solution could be determined with a longitudinal simulation of all variants. [3] consider three discrete power ratings for a parallel hybrid vehicle to optimise the control strategy first. Subsequently, the battery capacity, the power of the electrical machine, and the final drive ratio are 
optimised using the parameters of the optimised control strategy.

A continuous approach for the comparison of variants is used by [14]. The ICE, EM and battery size are continuously scalable and three powertrain configurations have been investigated. A genetic algorithm is used to optimise the powertrains on three cycles. The travelled distance is increased by raising the number of cycle-passes. This leads to an increased weight for BEV due to the battery size. Thus, the BEV is excluded because of a defined weight restriction although the carbon dioxide emissions could be lower.

Different driving performance demands based on load cycles are analysed for the manoeuvre-based dimensioning. The components are scaled to met the objectives based on the obtained data. The driving performance demands can be taken from standards [6] or can be extracted from driving cycles. In [4], the demands declared in the standard EV America: hybrid electric vehicle (HEV) technical specifications [6] were taken for dimensioning of serial hybrid powertrain components. An acceleration from 0 to $60 \mathrm{mph}$ determines the power of the electrical traction machine. Some more manoeuvres are used to scale the generator and combustion engine. The method proposed in [19] uses an acceleration from 0 to $100 \mathrm{~km} / \mathrm{h}$ and, additionally, a constant speed of $150 \mathrm{~km} / \mathrm{h}$ at $1 \%$ inclination of the road to derive the power and torque curve of a BEV. An extended method for the manoeuvre-based dimensioning for a plug-in hybrid vehicle in power-split configuration is provided in [15]. Besides the requirements from three standardised load cycles, the condition is formulated that the ICE should only be operated at load points with high efficiency. The result of the simulation is a hybrid powertrain with a more powerful EM than the ICE and a significant reduced energy consumption on the examined load cycles. To calculate the carbon dioxide emissions, the wellto-wheel emissions of the EM are neglected contrary to a holistic view on vehicle emissions.

All these approaches focus on one or a small number of propulsion systems and do not cover the variety of hybrid vehicle topologies that are examined in this paper. Furthermore, only standardised cycles have been the bases for dimensioning of the powertrains. A new approach in [5] focuses on user-based load cycles. Based on a large user profile database, a generation of cycles through the use of key parameters like energy balance and duration is performed. These cycles represent customer behaviour for a class of users. The proposed method in this paper focuses on the usage of the vehicle by an individual user and, therefore, real driving cycles are investigated to dimension the powertrain components. This paper presents a method to obtain an optimal propulsion system on usage-based load cycles using an optimisation of vehicle-specific parameters with a longitudinal dynamic simulation for different types of propulsion systems. The method uses recorded user profiles which were obtained in the metropolitan area of Hamburg, Germany. These profiles reflect a variety of typical usage behaviour from urban to motorway driving.

An overview of different types of propulsion systems is given in Sect. 2. In the third section, the considered usagebased load cycles are described. The simulation model and the mathematical description of a characteristic map are introduced in Sects. 4 and 5. The constrained nonlinear optimisation problem is formulated in Sect. 6. The results are given in Sect. 7. The conclusions of this paper are presented in Sect. 8.

\section{Types of propulsion systems}

A categorisation of passenger car propulsion systems can be conducted into three main groups as shown in Fig. 1. Conventional systems are internal combustion engines (ICE) consisting of diesel and petrol engines, which are frequently equipped with a turbocharger to get an efficiency improvement using the enthalpy within exhaust gases. Approx. 99\% of all new car registrations in the USA, Japan, China and Germany are covered by this group [8]. Vehicles with an electric machine as traction power engine form the group of battery electric vehicles (BEV). In passenger cars, mainly asynchronous induction machines (AIM) and permanent magnet synchronous machines (PMSM) are used as electric machines. PMSM have a higher power density and higher efficiency up to moderate speeds compared to AIM. Due to the use of rare earth metals, the application of PMSM leads to higher costs.

Hybrid powertrains are propulsion systems with at least two energy converters and two different energy storage systems [17, 20]. These can be categorised into series, parallel, series-parallel and power-split hybrid powertrains [13]. Figure 1 shows exemplary structures out of a vast number of possible configurations [10].

The advantages and disadvantages of these configurations are discussed in detail in the literature $[10,16]$. This paper focuses on plug-in hybrids with an installed electric power which allows to perform driving tasks in a wide area and over a distance which exceeds the typical driving distance in Germany of approx. $20 \mathrm{~km}$ [1]. The hybrid powertrain configurations, which are investigated, are series, parallel and power-split hybrids combined with petrol and diesel engines. This results in six propulsion systems for the hybrid group. Additionally, petrol, diesel , and battery electric vehicles are examined. In total, nine propulsion systems are optimised for minimal $\mathrm{CO}_{2}$ emissions on a specific use case. 
Fig. 1 Powertrain configurations for rear-wheel driven vehicles
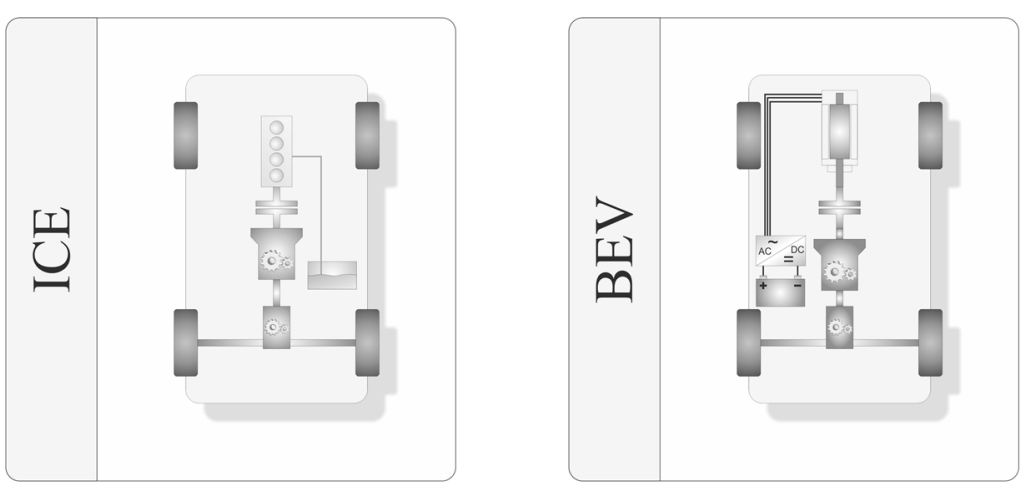

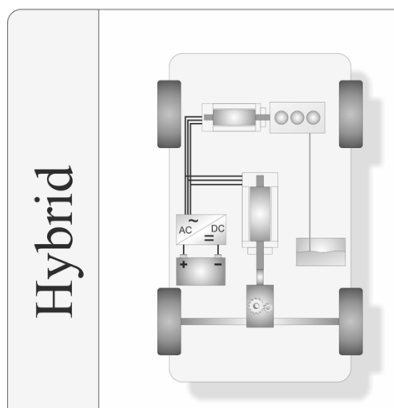

a) Series

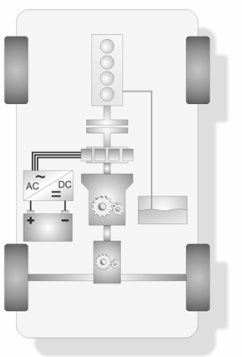

b) Parallel

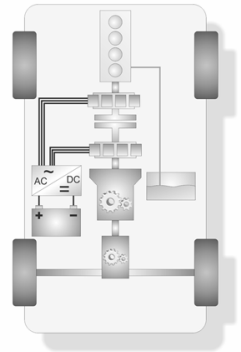

c) Series-Parallel

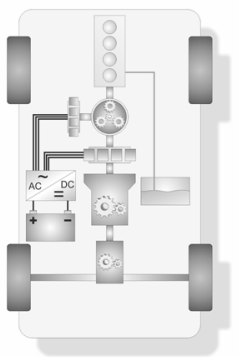

d) Power-Split

\begin{tabular}{|c|c|c|c|c|c|c|c|}
\hline \multirow{2}{*}{ 를 } & ICE & EM & Battery & Tank & $\begin{array}{c}\text { Power } \\
\text { electronics }\end{array}$ & Clutch & Gearbox \\
\hline & 0000 & 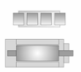 & +-1 & $\square$ & $A C^{\tilde{C}}=\mathrm{DC}$ & 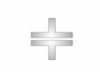 & 0 \\
\hline
\end{tabular}

\section{Load cycles}

Load cycles describe the usage of a vehicle and can be obtained by analysing a travelled route regarding speed and topology (elevation profile). Power and energy demand are calculated by analysing vehicle data and are unique for a specific use case. In other papers, mostly standardised load cycles for the optimisation of powertrains have been investigated like the new European driving cycle (NEDC), FTP-75 (USA), JC08 (Japan) or Artemis. Besides the Artemis cycle, all mentioned test cases are relevant for the type approval of vehicles and, therefore, the normative guideline for the design of powertrains with regard to fuel/ energy consumption and tailpipe emissions. Naturally, the customer or user will not achieve the prospected energy consumption and, accordingly, real emissions will be higher due to a different driving behaviour and load cycle. If the goal is to minimise vehicle emissions, then an optimised powertrain for every use case leads to minimised emissions for the whole transport sector.

In this paper, the powertrain will be fitted to user profiles which were measured using a gyro system. The motion data deliver the speed profile and GPS data are used for the elevation profile. An example for a measured urban driving profile is given in Fig. 2. Numerous stops and an average maximum speed slightly above $50 \mathrm{~km} / \mathrm{h}$ characterise this profile. The performed measurements cover a bandwidth of solely urban, extra urban and motorway driving.

The results of the optimisation will be presented by the example of two profiles. The first route is an urban driving profile as depicted in Fig. 2. The second route is mainly a motorway profile. Table 1 illustrates characteristics of profiles by presenting key parameters. The travelled distances $s$ and also the average $\bar{v}$ and maximum speed $v_{\max }$ increase from urban to motorway profile, while the stop times $t(v=0)$ decrease. A differentiated consideration has to be done for the acceleration. The maximum acceleration $a_{\max }$ decreases from urban to motorway cycle. This cannot be stated in general, because this value $a_{\max }$ depends to a large extent on the user and present traffic situations at the time of measurement and may differ substantially. The same holds for the minimum acceleration $a_{\min }$. Both profiles have been measured at least three times to eliminate random and disturbing events.

\subsection{Proportion variation of profiles}

In addition to the optimisation on a specific usage profile, an analysis with a continuously variation from $100 \%$ urban to $100 \%$ motorway driving was performed. This leads to a 

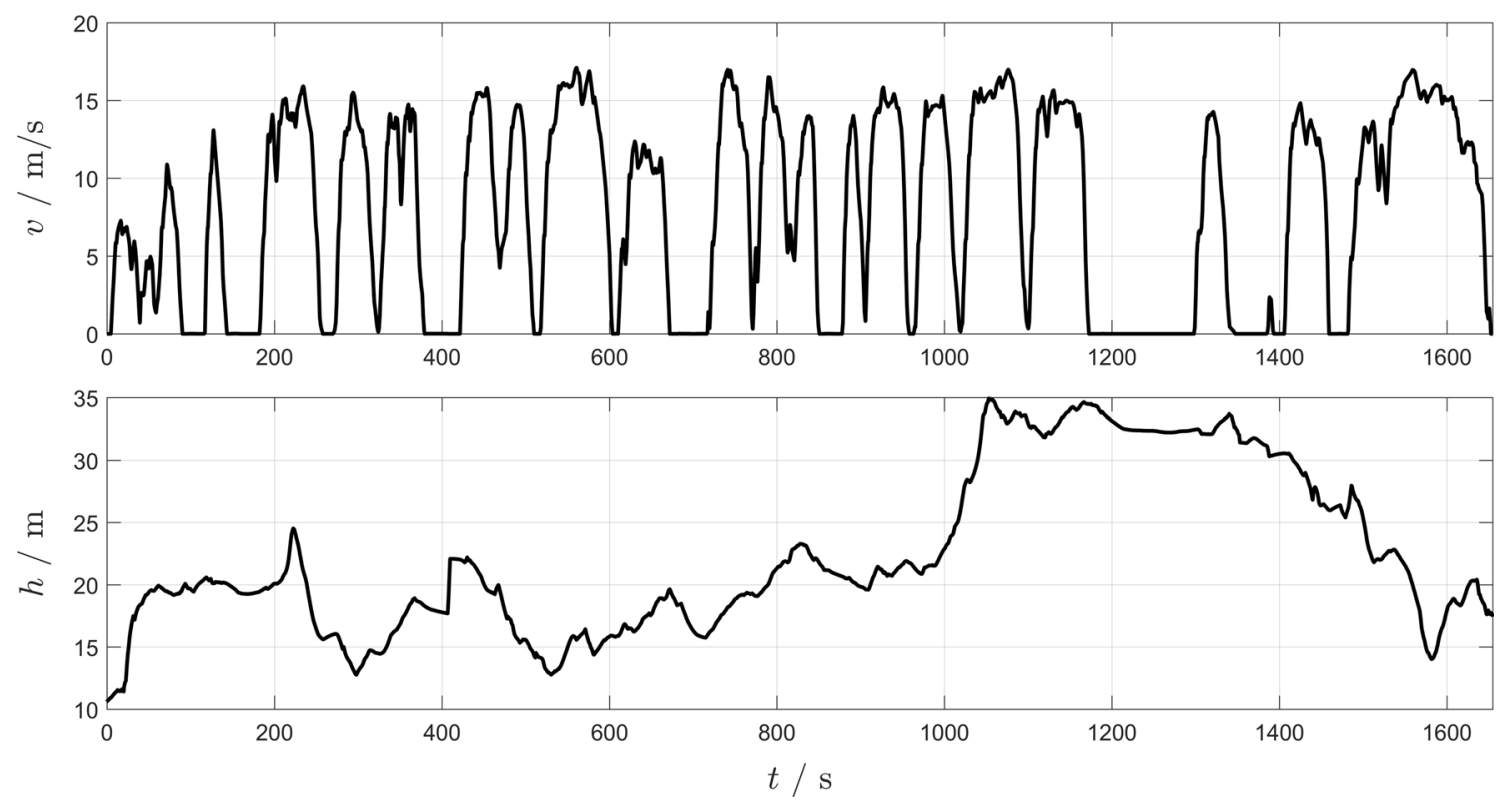

Fig. 2 Example for a measured urban driving speed and height profile

Table 1 Key parameter of the selected profiles

\begin{tabular}{lllllll}
\hline Route & $\mathrm{s} / \mathrm{km}$ & $v_{\max } / \mathrm{km} / \mathrm{h}$ & $\bar{v} / \mathrm{km} / \mathrm{h}$ & $a_{\max } / \mathrm{m} / \mathrm{s}^{2}$ & $a_{\min } / \mathrm{m} / \mathrm{s}^{2}$ & $t(v=0) / \mathrm{s}$ \\
\hline Urban & 12.4 & 61.5 & 28.4 & 3.96 & -3.99 & 261 \\
Motorway & 55.7 & 131.1 & 79.1 & 3.27 & -2.87 & 74 \\
\hline
\end{tabular}

more general view on the aspect which powertrain meets different driving profiles best. Therefore, a representative urban and an appropriate motorway profile have been extracted. The profiles are taken from single measurements and the characteristics regarding average speed, acceleration and stop times are fulfilled within a tolerance range from the mean value over all considered measurements for the urban and motorway profiles.

To improve the generalisability of the analysis, the combined travelled distance is set to approx. $19-20 \mathrm{~km}$ as this is half of the average daily travelled distance in Germany [1]. The travelled distance differs slightly between the varied profiles due to the fact that always a stop is needed at the end of the first part of the composite profile to secure a continuous transition from urban to motorway profile. When the desired portion of the urban profile is reached, the speed according to the speed profile is normally not zero at this time. An algorithm is applied which searches for the next and last stop in the profile. The stop with the smaller difference to the desired distance is chosen. For the motorway profile, a standardised stop procedure is applied which secures the compliance to the desired distance. To reduce optimisation time, the variation was performed in 30 discrete steps from $0 \%$ urban driving up to $100 \%$ urban driving.
Figure 3 shows three speed profiles of the variation in an exemplary manner. The $100 \%$ motorway-like profile in the top diagram shows approx. one and a half repetitions of the motorway profile with a total duration of $1060 \mathrm{~s}$ to reach the desired length of $20 \mathrm{~km}$. Due to the lower average speeds, the duration increases with higher percentage of urban driving (cf. middle and bottom diagram)

\section{Simulation model}

There are two principles how to build a longitudinal dynamic simulation depicted in Fig. 4. Depending on the application, these modelling methods have different advantages and disadvantages. The forward simulation is characterised by the application of a driver model which follows a given speed trajectory with the goal to minimise the speed error. Different driver models, e.g. a cautious driver or an aggressive driver, can be implemented for an investigation of their influence to vehicle behaviour. These models are complex with respect to the necessary modelling of human behaviour and parameter identification. For prompt reaction of the driver model and good quality of control, the forward simulation needs a small time step (in the sense of time-integration of the governing ordinary 

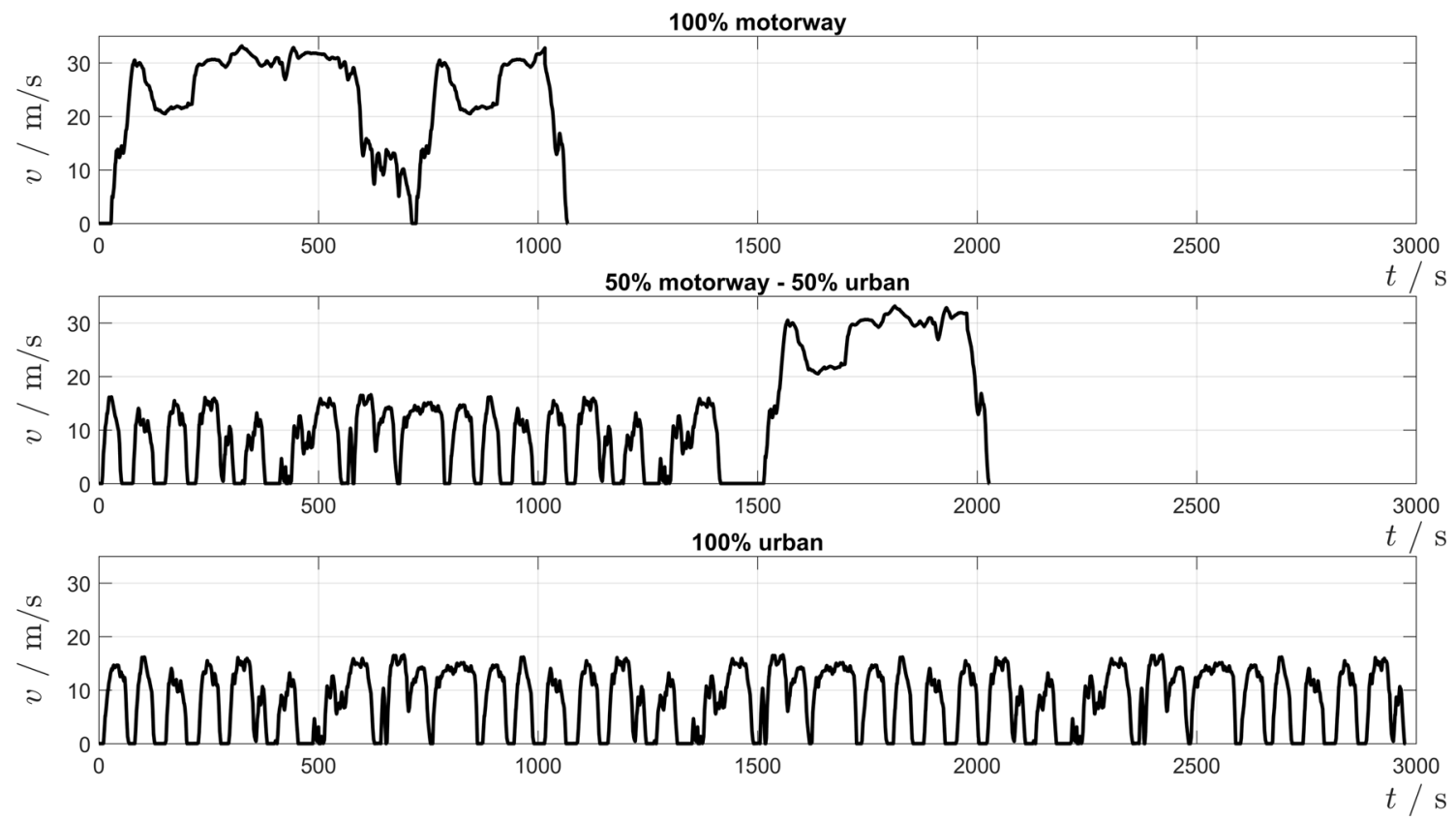

Fig. 3 Speed over time profiles for the proportion variation of urban and motorway cycles

Fig. 4 Principle structures for longitudinal simulation model, a forward and $\mathbf{b}$ backward simulation

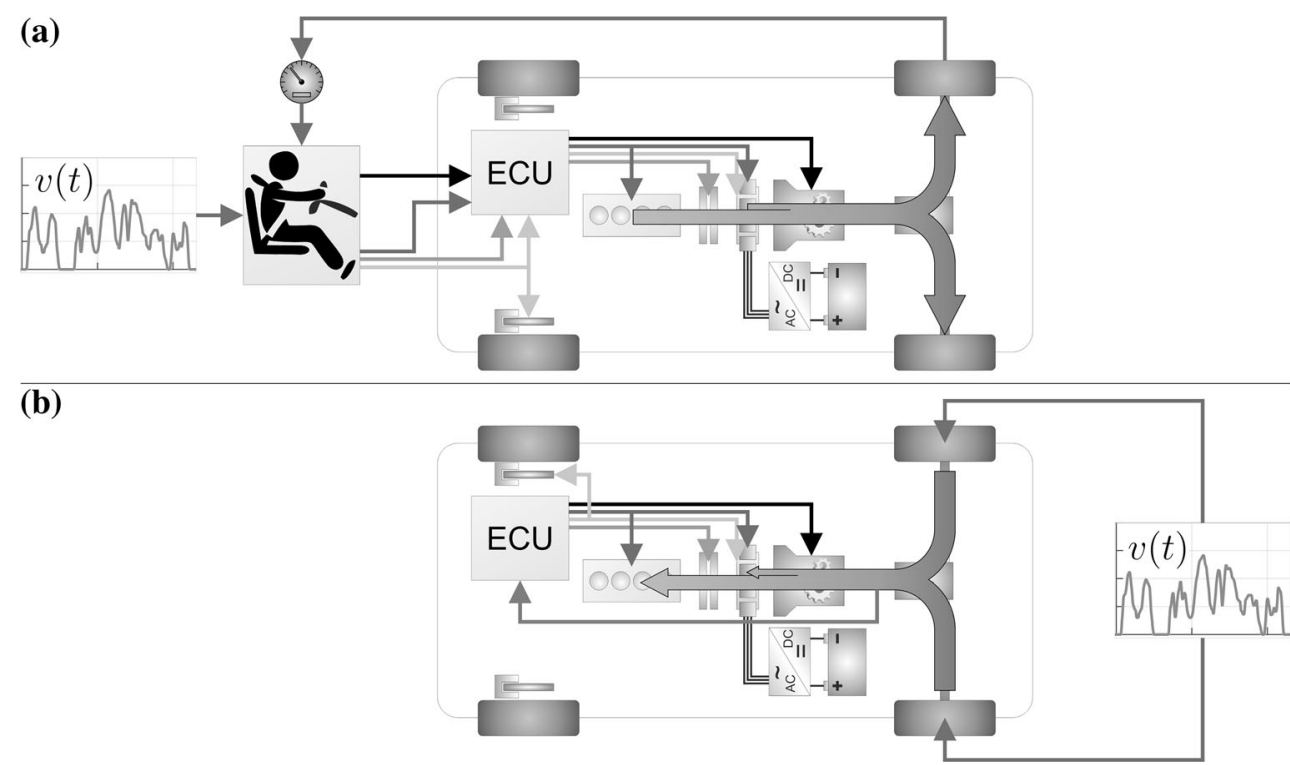

differential equations). This results in a long computation time.

However, backward simulations reverse the physical direction of action, as the traction machine loads are calculated backwards from the road loads to the engine loads. Solving the equation of motion (one second-order ordinary differential equation) for the vehicle, the load distribution for hybrid vehicles can be calculated in consideration of the operation strategy. With a backward simulation a longer time step of approx. $1 \mathrm{~s}$ can be chosen with the effect that the computation time decreases. The difference between the computation time of a forward and a backward simulation for a speed profile with a duration in the range of $25 \mathrm{~min}$ is approx. $16 \mathrm{~s}$ on a modern PC. For the desired application, the user-based speed profile implicitly contains the driving behaviour. Additionally, the simulation is used within an optimisation which requires short run times. In consequence, a backward simulation is chosen for the optimisation.

A detailed description of the simulation model is given in [9]. The following description gives a rough overview how the simulation works and where the optimisation affects the simulation parameters. 
The simulation model consists of three main systems: the vehicle system, the environment system and the operation strategy system. The environment system consists of the profile determining the state of driving and the calculation of the driving resistances. The result is the power demand at the wheels. The vehicle system consists of the brake system, the propulsion systems and the gearbox. The maps for fuel consumption and efficiency of the traction machines are part of the vehicle system. Thus, the maps can be varied during optimisation runs. The time-dependent output of the maps is saved and used as optimisation target. The approach of how the maps are derived will be discussed in Sect. 5.

\subsection{Validation of the simulation model}

The validation process for the simulation model is shown in Fig. 5. The coast down tests were performed with three different compact cars over a speed range from $v_{\mathrm{veh}}=100-30 \mathrm{~km} / \mathrm{h}$ following the regulations in [20]. The determination of the driving resistances polynomial coefficients has been derived using a least square optimisation as stated in Eq. 1.

$\sum_{i=1}^{n}\left(v_{s}\left(t_{i}, \alpha_{j}\right)-v_{m, i}\right)^{2}=\left\|\mathbf{v}_{s}-\mathbf{v}_{m}\right\|_{2}^{2}=\min$.

The simulated time-dependent speed $v_{s}$ for a coast down test is matched to the measured data $v_{m}$ by fitting the coefficients $\alpha_{j}$ so that the squared error is minimised. This method focuses on the validation of vehicle parameters like rolling resistance and drag coefficient. The inertial forces of the powertrain are partially considered as the clutch is disengaged and the gear lever is moved to neutral during the measurement. The result is a validated simulation model regarding the vehicle parameters without parts of the powertrain. Therefore, it has to be taken into account that parts of the powertrain (the rotating masses of these parts) cannot be disconnected and the measurement data include these inertias. This leads to a small error in the simulation in favour of a modular vehicle with an interchangeable powertrain. The different weight and inertia of the components affect the driving resistance [13] and consequential the coast down behaviour. The simulation takes this influence into account merely the rolling resistance change is neglected.

\section{Modelling characteristic maps}

A characteristic map within the scope of this paper describes the efficiency of a machine subject to two independent variables. It is focused on electric and combustion engines. Therefore, efficiency maps for EM's and brakespecific fuel consumption maps (BSFC) for ICE will be considered. The efficiency map for an ICE can be derived by calculating the reciprocal of the product of specific fuel consumption and calorific value. The basis for further considerations constitutes the BSFC's as they are more common.

The optimal propulsion system for a specific usage can only be determined using a dimensioning method that is able to scale and shift the maps in a way that the integral over all load points delivers minimum fuel consumption and maximum efficiency, respectively. To reduce the complexity of the optimisation problem, a simple mathematical description is needed. For the proposed method, a quadric surface in $\mathbb{R}^{3}$ models the characteristic maps, which is represented by the general formula:

$$
\begin{aligned}
Q(x)= & \sum_{i=1}^{3} \sum_{j=1}^{3} a_{i j} x_{i} x_{j}+\sum_{i=1}^{3} b_{i} x_{i}+c=\underline{\mathrm{x}^{T}} \underline{\underline{\mathrm{A}}} \underline{\mathrm{x}} \\
& +\underline{\mathrm{b}^{T}} \underline{\mathrm{x}}+c=0 .
\end{aligned}
$$

The tuple $\underline{\mathrm{X}}=\left(x_{1}, x_{2}, x_{3}\right)$ describes the spatial directions; tuple $\underline{\mathrm{b}}$ and matrix $\mathrm{A}$ contain the shaping coefficients for the linear and quadratic terms, respectively. As there are many different forms of 3-dimensional hyper surfaces, an elliptic paraboloid turns out to meet the requirements best for modelling characteristic maps. The normal form of this quadric is

$\frac{x_{1}^{2}}{\gamma_{1}^{2}}+\frac{x_{2}^{2}}{\gamma_{2}^{2}}-\frac{x_{3}}{\gamma_{3}}=0$.

For this specific use, the three variables $x_{i}, i=1, \ldots, 3$ are the rotational speed $n$, the output torque $M$ and the specific fuel consumption be (efficiency $\eta$ ) of the engine. The three

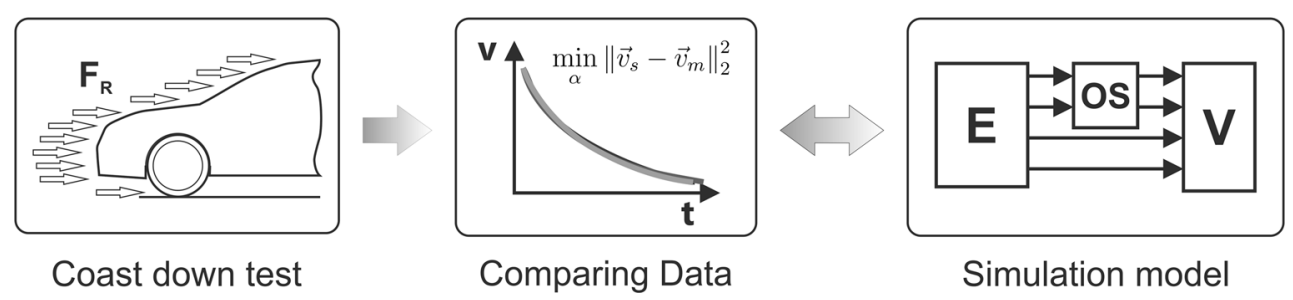

Fig. 5 Validation process of the simulation model using coastdown tests 
free parameters in Eq. $3 \gamma_{i}, i=1, \ldots, 3$ only permit shape modifications. To extend the degree of freedom, additional parameters for shifting and rotation of the paraboloid are introduced. These allow a free movement and spatial location of the surface. The related equation in matrix notation reads as follows:

$\tilde{Q}(x)=\underline{\tilde{\mathrm{x}}}^{T} \underline{\underline{\tilde{\mathrm{A}}}} \underline{\tilde{\mathrm{x}}}+\underline{\tilde{\mathrm{b}}}^{T} \underline{\tilde{\mathrm{x}}}=0$,

where

$\underline{\tilde{\mathbf{x}}}=\underline{\underline{\mathrm{D}}}_{x_{2}} \underline{\underline{\mathrm{D}}}_{x_{1}} \underline{\underline{\mathrm{D}}}_{x_{3}}(\underline{\mathrm{x}}-\underline{\mathrm{d}})$.

$\underline{\mathrm{D}}_{x_{i}}, i=1, \cdots, 3$ denote rotation matrices of the form

$$
\begin{aligned}
& \underline{\underline{\mathrm{D}}}_{x_{1}}=\left(\begin{array}{ccc}
1 & 0 & 0 \\
0 & \cos (\varphi) & -\sin (\varphi) \\
0 & \sin (\varphi) & \cos (\varphi)
\end{array}\right), \\
& \underline{\underline{\mathrm{D}}}_{x_{2}}=\left(\begin{array}{ccc}
\cos (\vartheta) & 0 & \sin (\vartheta) \\
0 & 1 & 0 \\
-\sin (\vartheta) & 0 & \cos (\vartheta)
\end{array}\right), \\
& \underline{\underline{\mathrm{D}}}_{x_{3}}=\left(\begin{array}{ccc}
\cos (\psi) & -\sin (\psi) & 0 \\
\sin (\psi) & \cos (\psi) & 0 \\
0 & 0 & 1
\end{array}\right),
\end{aligned}
$$

and the tuple $\underline{d}$ describes the translation. Thus, Eq. 4 has nine free parameters (three angles of rotation, three translation parameters, and three shaping parameters $\gamma_{1}, \gamma_{2}, \gamma_{3}$ ) which can be varied to fit the surface to a specific usage. Within an optimisation algorithm, reasonable restrictions for the parameters have to be applied to ensure the elliptic paraboloid still represents a characteristic map. To obtain these boundary values and to validate this method of abstraction, the quadric is fitted to a real characteristic map of a combustion engine by the formulation of a least-square problem of the form:

$\min \left\|r_{b e}\right\|_{2}^{2}=\min \left\|b e_{n, p}-b e_{n, k}\right\|_{2}^{2}$,

where $b e_{n, k}$ is the standardised real characteristic map and $b e_{n, p}$ is the standardised paraboloid. The formulation of the least square problem for an EM is obtained by replacing $b e_{n, p}$ and $b e_{n, k}$ by $\eta_{p}$ and $\eta_{k}$. Solving Eq. (6) under the constraint that the solution is always in the range $\underline{\tilde{\mathbf{x}}}_{l b} \leq \underline{\tilde{\mathrm{x}}}<\underline{\tilde{\mathrm{x}}}_{u b}$ (where $\underline{\tilde{\mathrm{x}}}_{l b}$ and $\underline{\tilde{\mathrm{x}}}_{l b}$ are the lower and upper boundary tuples), the obtained hyper surface is a representative for the real characteristic map. The minimisation problem is formulated over a limited area of rotational speed to improve accuracy of the results. This holds for ICE, because under normal conditions and for a normal driver the rotational speed of the engine will not exceed $60 \%$ of the maximum engine speed. A differentiated consideration has to be done for EM. Hybrids with an EM arranged coaxial to the ICE on the same shaft have to be treated as stated above. When the EM has a direct connection to the wheels, the speed range is normally fully exploited and the least-square problem has to be formulated over the full speed range. Figure 6 shows two fittings of the paraboloid.

Comparing a simulation run for a petrol driven compact car with a fitted paraboloid as characteristic map on the new European driving cycle (NEDC) [20] with the manufacturer data, the deviation is approx $4 \%$. Performing the same test for a parallel hybrid, the deviation in electric energy consumption is about $3 \%$ and for the ICE an error of approx. 5\% is reached. This shows that using an elliptic paraboloid as representative for specific fuel consumption maps of an ICE and efficiency maps of an EM is an appropriate method to model the operating behaviour of these engines.

Building on the explanations in Sect. 4 in conjunction with the results stated above, the simulation model as well as the abstraction of characteristic maps can be considered as validated. The next section deals with the optimisation (i.e. the minimisation of the $\mathrm{CO}_{2}$ emissions) of the powertrain for the validated vehicle model.

\section{Definition of the optimisation problem}

To examine various powertrain configurations and their comparison, an assessment procedure applicable to all configurations has to be established. Due to different energy conversion processes for ICE and EM, the considered system boundaries affect the comparison result. Furthermore, the comparison value has to be determined. As the efficiency of an ICE driven vehicle is rated by the fuel consumption and the efficiency of an EM driven vehicle by electrical energy consumption, these two reference values have to be comparably summarised. The carbon dioxide emissions are the basic parameter for the consideration of these two propulsion engines. For the choice of system boundaries, the tank-to-wheel emissions neglect the production chain for both systems and, additionally, the tankto-wheel emissions privilege EM due to the non-existent local emissions. In a global consideration, the well-towheel analysis is the only reasonable approach, as overall costs measured by $\mathrm{CO}_{2}$ emissions are taken into account.

The optimisation problem for the elliptic paraboloid (see Eq. 4) has nine free parameters in a nonlinear multi variable function. The application of a gradient method for the optimisation is possible due to the continuously differentiable function. The constrained nonlinear programming problem has the form:

$$
\begin{aligned}
& \min _{\underline{x}} \mathrm{CO}_{2}(\underline{\mathrm{x}}) \\
& \text { over } \underline{\mathrm{x}} \in \mathbb{R}^{n}
\end{aligned}
$$



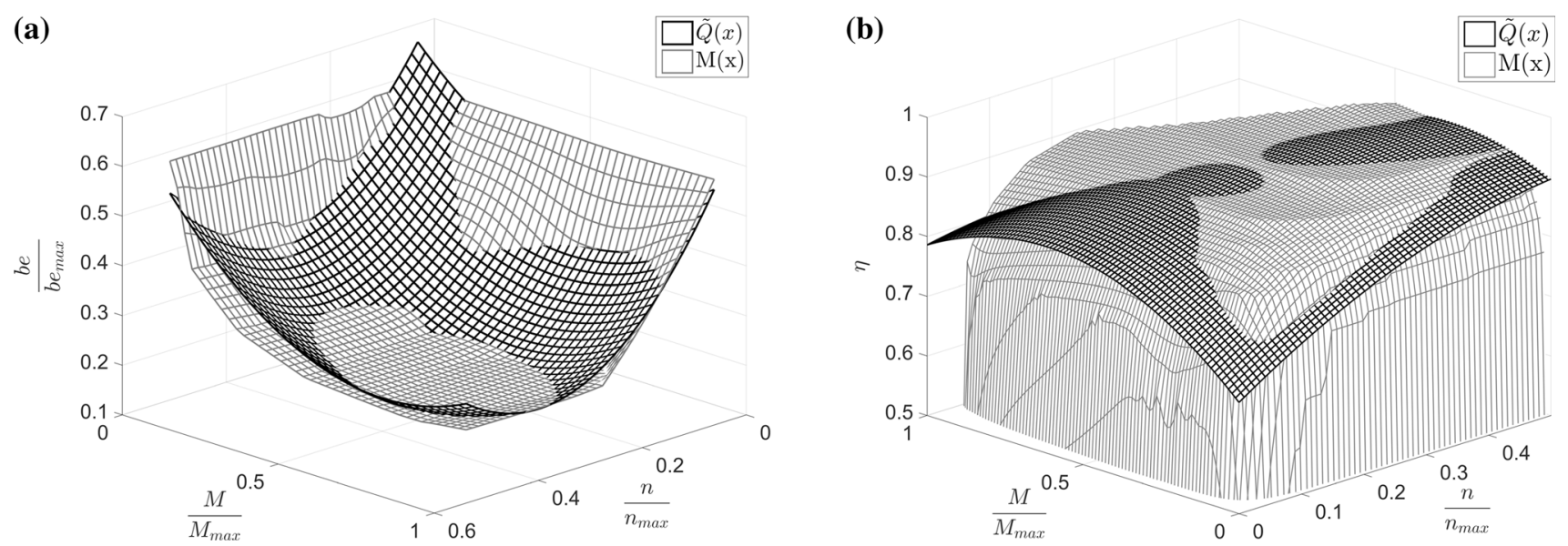

Fig. 6 Least-square problem results for an ICE and an EM characteristic map

$$
\text { subject to } \underline{\mathrm{X}} l b \leq \underline{\mathrm{X}}<\underline{\mathrm{X}} u b \text {. }
$$

where $\mathrm{CO}_{2}(\underline{\mathrm{x}})$ is the well-to-wheel equivalent of the energy and fuel consumption of the vehicle. The free parameters are restricted by the boundary condition tuples $\underline{\mathrm{X}}_{l b}$ and $\underline{\mathrm{X}}_{u b}$. This preserves the shape of the optimised elliptic paraboloid and the limitation of parameter space.

For the variation of the parameters in $\mathrm{x}$, an interiorpoint-method is chosen. The objective function of the optimisation problem is formed by the $\mathrm{CO}_{2}$ emissions, which have to be minimised. The argument of the objective function is the parameters of the elliptic paraboloid or elliptic paraboloids for hybrid vehicles. The $\mathrm{CO}_{2}$ emissions for a usage profile are derived using the starting parameter which are used to form the characteristic maps within the longitudinal simulation model. The determined emissions form the function value at location $\underline{\mathrm{x}}_{i}$. By solving the linear equation system

$\left.\underline{\nabla^{2}} \mathrm{CO}_{2}\right|_{\underline{\mathrm{x}}_{=} \underline{\mathrm{x}}_{i}} \Delta \underline{\mathrm{x}}_{i}=-\left.\underline{\nabla} \mathrm{CO}_{2}\right|_{\underline{\mathrm{x}}=\underline{\mathrm{x}}_{i}}$

the correction tuple $\Delta \underline{\mathrm{x}}_{i}$ is determined. This tuple provides in combination with $\underline{\mathrm{X}}_{i}$ the parameter for the next iteration step:

$\underline{\mathrm{X}}_{i+1}=\underline{\mathrm{x}}_{i}+\Delta \underline{\mathrm{X}}_{i}$.

The iteration repeats until the abort criterion is fulfilled:

$\Delta \mathrm{CO}_{2}=\left|\mathrm{CO}_{2}\left(\underline{\mathrm{x}}_{i+1}\right)-\mathrm{CO}_{2}\left(\underline{\mathrm{x}}_{i}\right)\right| \leq \varepsilon$.

For hybrid vehicles, the parameter tuple $\underline{\mathrm{x}}$ is extended for two or more engines. As the goal is to minimise the total $\mathrm{CO}_{2}$ emissions, a variable power distribution between electric and fuel driven machine is necessary, which is achieved by the joint reflection within the optimisation problem. In this paper, the propulsion systems mentioned in Sect. 2 are investigated. For each system, the optimisation problem has to be solved and subsequently the comparison of the optimisation results leads to the optimal propulsion system for the considered use case. Exemplary results are shown in the next section.

\section{Optimisation results}

First of all, the illustration of the results will be exemplarily given for two different usage profiles. The key parameters of the selected profiles are stated in Sect. 3 Table 1. Then, in Sect. 7.1, the combined motorway-urban profiles are investigated. Additionally, in Sect. 7.2, a consideration of the influence of an additional devices, e.g. the air conditioning, is performed.

The optimisation result of a PHEV on the motorway profile is exemplary shown in Fig. 7. Two different states are depicted in the torque over speed diagrams for the traction engines. The initial state is represented by grey coloured graphs, and the description of the optimised propulsion system is shown by the black coloured graphs. Additionally, the load points are shown by black circles. For the representation of the elliptic paraboloid, i.e. the characteristic map, a plot of contour lines shows the initial and optimised state coloured in the same manner as mentioned above.

The examined plug-in parallel hybrid vehicle is fully charged at the beginning of the cycle. This boundary condition holds for all further considerations. Another boundary condition for the torque curve is that all load points should be covered with the respective traction machine, which prevents the algorithm to further decrease the torque level. The distribution of the load points is owed to the operation strategy. At the beginning of the cycle, the electric machine is able to cover all load requirements. With progressive depletion of the battery, the ICE takes over the load and the electric machine is only used for 

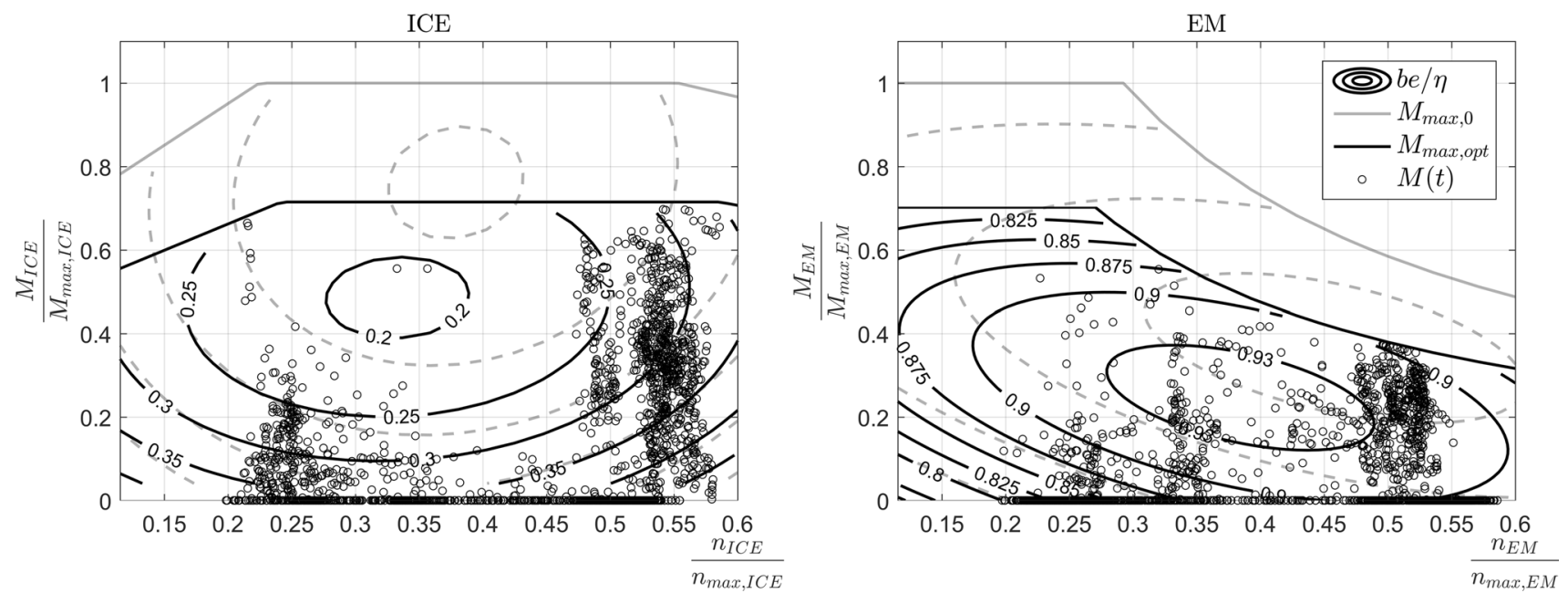

Fig. 7 Characteristic maps of an PHEV before and after the optimisation

recuperation. The amount of recuperated energy in this motorway cycle is not high enough so that the EM is not able to cover the short urban driving at the end of the cycle.

In Fig. 8, the speed and both the fuel and energy consumption for the motorway cycle are shown. This profile is characterised by a long motorway turn in the middle of the cycle and short urban sections at the beginning and the end. The Battery is rapidly depleting on the motorway. At $1100 \mathrm{~s}$ the ICE takes over for the rest of the cycle. This forces the optimisation algorithm to rate the ICE and the $\mathrm{EM}$ at the same level, as both have to fulfil motorway driving with high power demand. The $\mathrm{CO}_{2}$ emission for this cycle is $\mathrm{CO}_{2, \text { phev }}=153 \mathrm{gCO}_{2} / \mathrm{km}$ in total. The results for the other investigated propulsion systems are shown in Table 2. For the calculation of the well-to-wheel emissions, an emission factor of $e_{\mathrm{el}}=562 \mathrm{gCO}_{2} / \mathrm{kWh}$ is set for the electric energy production which corresponds to the emission factor in Germany in 2014 [11]. The factor for the carbon dioxide emissions of the ICE is $e_{\text {petrol }} \approx$ $309 \mathrm{gCO}_{2} / \mathrm{kWh}$ for petrol engines and $e_{\text {diesel }} \approx$ $303 \mathrm{gCO}_{2} / \mathrm{kWh}$ for diesel engines [7]. To derive the overall $\mathrm{CO}_{2}$ emissions, the factors $e_{i}$ are multiplied with the energy consumption or rather with the fuel consumption multiplied by the energy content of the considered fuel.

The carbon dioxide emissions for the optimised vehicles show a broad range with the highest emissions nearly twice as high as the lowest. This one is achieved by the Diesel driven vehicle with $119 \mathrm{gCO}_{2} / \mathrm{km}$. The second lowest emissions are produced by the BEV which shows that with
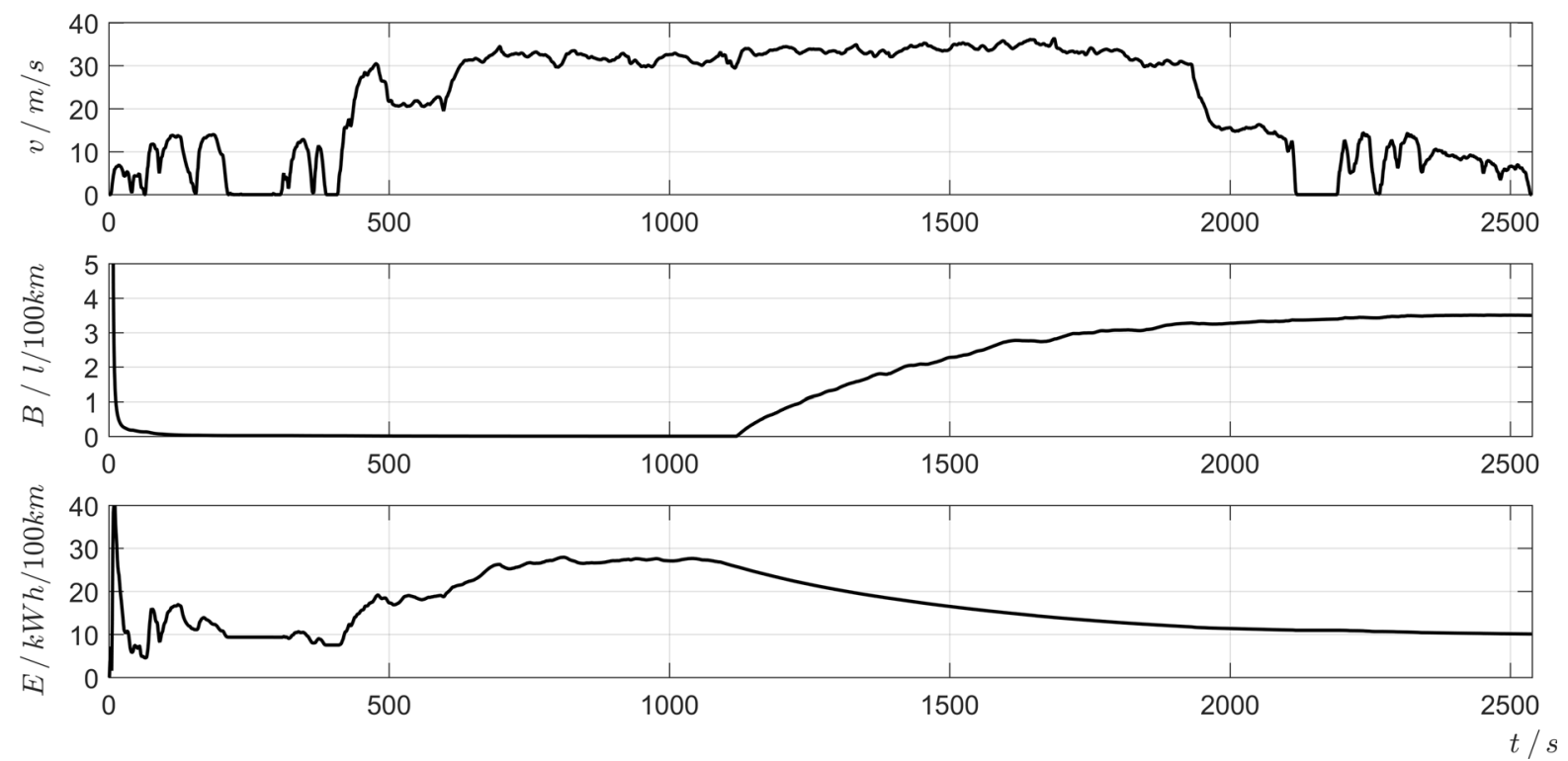

Fig. 8 Speed, fuel and energy consumption graphs on motorway cycle for PHEV 
Table 2 Results for the optimisation on motorway cycle

\begin{tabular}{llll}
\hline Prop.-System & $M / \mathrm{gCO}_{2} / \mathrm{km}$ & $B / 1 / 100 \mathrm{~km}$ & $E / \mathrm{kWh} / 100 \mathrm{~km}$ \\
\hline Petrol & 160 & 5.8 & - \\
Diesel & 119 & 3.9 & - \\
PSHEV & 193 & 6.2 & 3.8 \\
PSHEV-D & 194 & 5.7 & 6.8 \\
PHEV & 153 & 3.5 & 10.2 \\
PHEV-D & 143 & 3.2 & 10.0 \\
SHEV & 225 & 2.6 & 27.4 \\
SHEV-D & 219 & 2.2 & 27.4 \\
BEV & 132 & - & 23.5
\end{tabular}

a lower emission factor or the use of sustainable generated energy the BEV could even deliver the lowest emissions. For the SHEV vehicle, the high energy consumption is the result of a higher battery capacity compared to the PHEV. While the PHEV drives nearly half of the profile with the ICE, the SHEV uses the EM for the majority of the cycle. The high energy demand during motorway driving causes this high energy consumption.

For the urban cycle, the results are shown in Table 3. As the power demand is distinctly lower under urban driving conditions, the parallel and serial hybrids complete this profile without using the ICE. In combination with a shorter travelled distance of $12.4 \mathrm{~km}$, the battery capacity is sufficient to complete the cycle only electric driven. This leads to low $\mathrm{CO}_{2}$ emissions for the electric supported or purely electric driven vehicles, with the lowest emissions for the BEV.

\subsection{Variation of profiles and emission factor}

In Sect. 3.1, the variation of user profiles was introduced. This will be used in an investigation considering the variation of two independent variables. The first one is the proportion between urban and motorway driving with a bandwidth from $100 \%$ urban to $100 \%$ motorway driving. The second one is the emission factor which is used to calculate carbon dioxide emissions for the electric energy consumption. This factor is considered with a range from $5 \mathrm{gCO}_{2} / \mathrm{kWh}$ for solely sustainable generated electric energy to the current emission factor in Germany with $562 \mathrm{gCO}_{2} / \mathrm{kWh}$. To reduce optimisation runs, a discrete step of $10 \mathrm{gCO}_{2} / \mathrm{kWh}$ for the variation of emission factor has been chosen.

The combined variation of these factors leads to a Matrix with $30 \times 57$ entries. On each entry, optimisation runs for the nine investigated vehicles have to be performed. The results are shown in Fig. 9. It shows the percentage distribution between urban and motorway cycle on the axis of ordinate over the variation of the emission factor on the axis of abscissae. Additionally, the $\mathrm{CO}_{2}$ emissions are plotted as contour lines. The unsteady course of the contour lines results from the fact that a steady transition from urban to motorway profile is needed and from the elimination of recuperation phases in the motorway cycle. With the directive to keep the travelled distance at approx. $20 \mathrm{~km}$, the urban profile is cut at the point where the desired distance is reached. If the speed at this point is higher than zero, a hard cut would lead to a high deceleration at the transition to motorway cycle. To prevent this, the urban profile is prolonged to the next stop. In contrast, the motorway cycle is cut at the time step where the travelled distance is reached. This causes unsteadiness at some points due to an elimination of recuperation phases.
Table 3 Results for the optimisation on urban cycle

\begin{tabular}{llll}
\hline Prop.-System & $M / \mathrm{gCO}_{2} / \mathrm{km}$ & $B / 1 / 100 \mathrm{~km}$ & $E / \mathrm{kWh} / 100 \mathrm{~km}$ \\
\hline Petrol & 169 & 6.5 & - \\
Diesel & 111 & 3.7 & - \\
PSHEV & 151 & 3.2 & 11.3 \\
PSHEV-D & 135 & 2.6 & 11.1 \\
PHEV & 58 & - & 10.3 \\
PHEV-D & 77 & - & 13.6 \\
SHEV & 54 & - & 9.7 \\
SHEV-D & 59 & - & 10.5 \\
BEV & 47 & - & 8.4 \\
\hline
\end{tabular}




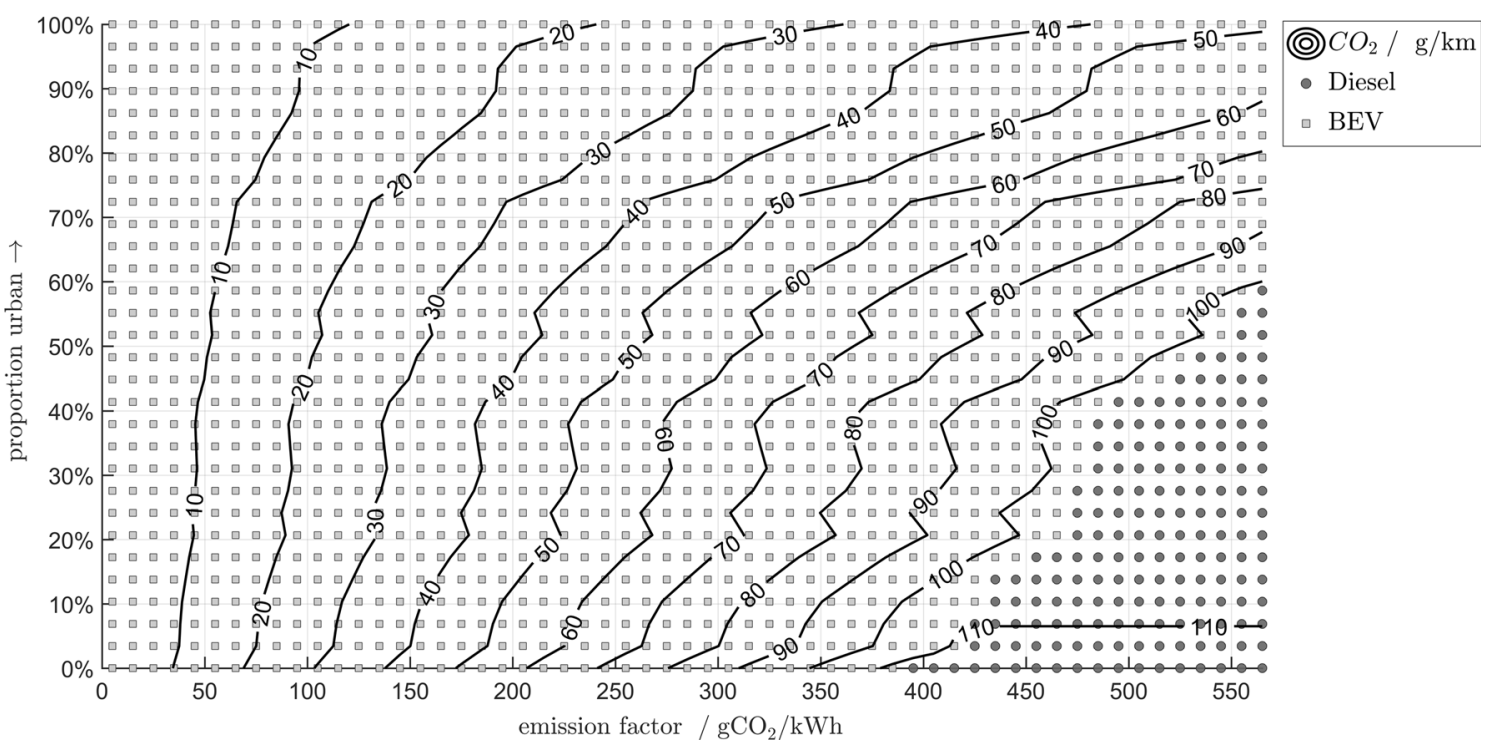

Fig. 9 Optimisation results for variation of speed profiles and emission factor

The lower right corner in Fig. 9 represents 100\% motorway driving with an emission factor of $562 \mathrm{gCO}_{2} / \mathrm{kWh}$. The upper left corner represents $100 \%$ urban driving over the same distance with an emission factor of $5 \mathrm{gCO}_{2} / \mathrm{kWh}$. The dots and squares indicate the optimal propulsion system in the sense of lowest carbon dioxide emissions at the considered point.

Remarkable is the absence of any hybrid vehicle as propulsion system with the lowest carbon dioxide emissions at any point. Only the BEV and Diesel driven vehicle are optimal in this investigation. The Diesel engine delivers the lowest $\mathrm{CO}_{2}$ emissions at $100 \%$ motorway driving up to an emission factor of approx. $400 \mathrm{gCO}_{2} / \mathrm{kWh}$. This shows the lower efficiency compared to the BEV. With identical energy consumption and efficiency, the break even point would have been at $e_{\text {diesel }} \approx 303 \mathrm{gCO}_{2} / \mathrm{kWh}$. The break even point shifted to a higher emission factor indicates the overall higher efficiency of the BEV. The course of the break even point over increasing urban driving proportion points towards higher emission factor due to the decreasing load of the traction machines. Lower loads lead to a decreasing efficiency considering the characteristic map of the diesel engine. The efficiency of the BEV suffers from the same effect but the percentage loss is significantly lower. At $60 \%$ urban driving and above, the BEV is the propulsion system with the lowest carbon dioxide emissions for all examined emission factors.

\subsection{Effect of additional loads}

Additional loads, e.g. heating or air-condition, in a passenger car can affect the energy consumption depending on the propulsion system in a significant way. On the one hand, electrical energy is inter alia needed for nowadays electrified auxiliary devices like an electrical water pump or steering and on the other hand thermal energy is needed for the heating of the passenger cabin. By the consideration of heating and cooling capacity, the conventional and alternative propulsion systems are affected in a different manner with an advantage for the conventional propulsion systems concerning the process waste heat of the ICE. A comparative analysis for the different propulsion systems is, therefore, performed for the air conditioning of the passenger cabin. The air conditioning means an additional load for both conventional and electric propulsion systems.

For a first investigation an additional, mean constant load of $3 \mathrm{~kW}$ for the air conditioning is assumed, although the power demand of the air conditioning compressor is higher at the beginning of the cooling process and lower in steady state [2]. The user profile used for the comparison is a combined urban and motorway profile with key parameters as shown in Table 4. This combined profile shows key parameters that are in between the urban and motorway profile as shown in Table 1. Remarkable are the maximum and minimum accelerations as they are indicating a different usage behaviour. The maximum acceleration $a_{\max }$ in the considered cycle is lower than the corresponding value of the motorway profile and the absolute value of the minimum acceleration $a_{\min }$ is higher than in the urban profile. This means that the driver has a conservative acceleration and a more progressive deceleration behaviour. Using this profile for two optimisation runs one without the additional load of $3 \mathrm{~kW}$ and one with the constant load applied, the results presented in Table 5 show a different behaviour depending on the considered energy converter. 
Table 4 Key parameter of the profile for the comparison of the effect of auxiliary users

\begin{tabular}{|c|c|c|c|c|c|c|}
\hline Route & $\mathrm{s} / \mathrm{km}$ & $v_{\max } / \mathrm{km} / \mathrm{h}$ & $\bar{v} / \mathrm{km} / \mathrm{h}$ & $a_{\max } / \mathrm{m} / \mathrm{s}^{2}$ & $a_{\min } / \mathrm{m} / \mathrm{s}^{2}$ & $t(v=0) / \mathrm{s}$ \\
\hline & 20.1 & 118.7 & 55.5 & 2.85 & -4.16 & 184 \\
\hline
\end{tabular}

Table 5 Comparison of the optimisation results on the combined profile without and with air conditioning

\begin{tabular}{llll}
\hline Prop.-System & $M_{n o A C} / \mathrm{gCO}_{2} / \mathrm{km}$ & $M_{A C} / \mathrm{gCO}_{2} / \mathrm{km}$ & $\Delta M / \%$ \\
\hline Petrol & 145 & 196 & +35 \\
Diesel & 93 & 123 & +32 \\
PSHEV & 126 & 180 & +43 \\
PSHEV-D & 153 & 218 & +42 \\
PHEV & 101 & 163 & +61 \\
PHEV-D & 106 & 167 & +58 \\
SHEV & 106 & 167 & +58 \\
SHEV-D & 107 & 168 & +57 \\
BEV & 94 & 149 & +59 \\
\hline
\end{tabular}

The column $M_{n o A C}$ lists the results without the load of the air condition and $M_{A C}$ the results with the additional $3 \mathrm{~kW}$ load. The last column $\Delta M$ shows the percentage change between the two load conditions. For the propulsion systems only driven by an ICE, the percentage change is in the range of $32-35 \%$. With increasing proportion of traction energy delivered by an EM, the percentage change raises. Those propulsion systems completing the profile with electrical driving only show a percentage change in the $\mathrm{CO}_{2}$ emissions of nearly $60 \%$. The different increases can be explained by an efficiency improvement of the ICE due to the additional load. By considering the characteristic map of an ICE, the additional load leads to a higher torque demand at the same engine speed and in consequence to a shift of the load points to regions with higher efficiency. The EM has an higher efficiency overall and the additional load leads only to a smaller change in the efficiency so that the efficiency gain is superimposed by the higher energy demand.

\section{Conclusions}

The proposed method allows the optimisation of powertrains for a specific usage-and in consequence the determination of an optimal powertrain for a user profile with respect to carbon dioxide emissions. Through the dimensioning of powertrain components, a decrease in $\mathrm{CO}_{2}$ emission is achieved. The emissions (as a result of a longitudinal dynamic simulation) form the objective function of the optimisation problem. Taking the well-to-wheel emissions into account, not only the local emissions of the vehicle but also the global emissions are investigated in this study.
The results show that the lowest $\mathrm{CO}_{2}$ emissions for an urban profile can be achieved with a battery electric vehicle. For a pure motorway cycle, the optimal propulsion system is a modified diesel engine. The variation of the load cycle between $100 \%$ urban and $100 \%$ motorway driving in combination with the variation of the emission factor delivers the result that (under consideration of the current emission factor in Germany) the conventional powertrain with a diesel engine is up to a proportion of $60 \%$ urban driving the system with the lowest $\mathrm{CO}_{2}$ emissions. Beyond this proportion, the BEV emits the lowest $\mathrm{CO}_{2}$ emissions.

The consideration of additional loads has been exemplary performed using an constant additional load. This should approximate driving with air conditioning. The comparison of the results for an combined urban and motorway profile shows a lower gain in $\mathrm{CO}_{2}$ emissions for conventional ICE propulsion systems due to an higher increase in efficiency. Further investigation for the heating and cooling of the passenger cabin will be performed with more detailed models to quantify the differences between the propulsion systems. Another approach can be taken through the consideration of biogene fuels which are nowadays to a small proportion part of the conventional fuels in Germany with the future perspective of a rising proportion. This leads to a similar investigation as the variation of the emission factor but with the $\mathrm{CO}_{2}$ emissions of the fuels as object of investigation.

The objective to determine an optimal powertrain for different usage profiles permits the inclusion of more parameters within further analyses. Through the analysis of the driving behaviour, characteristic values can be extracted which can be used within a multi criteria analyses. Furthermore, enhancements for the simulation model can be done with the inclusion of optimisation-based control strategies.

Open Access This article is distributed under the terms of the Creative Commons Attribution 4.0 International License (http://crea tivecommons.org/licenses/by/4.0/), which permits unrestricted use, distribution, and reproduction in any medium, provided you give appropriate credit to the original author(s) and the source, provide a link to the Creative Commons license, and indicate if changes were made.

\section{References}

1. Bertram, M., Bongard, S.: Electromobility in Motorised Private Transport. Springer Wiesbaden, Wiesbaden (2014) 
2. Braess, H.H., Seiffert, U.: Handbook of Automotive Engineering. SAE International, Wiesbaden (2005)

3. Buerger, S., Lohmann, B., Merz, M., Vogel-Heuser, B., Hallmannsegger, M.: Multi-objective optimization of hybrid electric vehicles considering fuel consumption and dynamic performance. In: Vehicle Power and Propulsion Conference (VPPC), 2010 IEEE, pp. 1-6 (2010). doi:10.1109/VPPC.2010.5729128

4. Chanda, S.: Powertrain sizing and energy usage adaptation strategy for plug-in hybrid electric vehicles. Master's thesis, University of Akron (2008). https://etd.ohiolink.edu/rws_etd/ document/get/akron1208385855/inline

5. Eghtessad, M.: Optimale Antriebsstrangkonfigurationen für Elektrofahrzeuge. Dissertation, Technische Universität Braunschweig, Braunschweig (2014)

6. Electric Transportation Applications: Ev america: Hybrid electric vehicle (hev) technical specifications (2005). http://energy.gov/ sites/prod/files/2015/04/f21/hev_techspecs_final.pdf

7. Ernst, C.S.: Energetische, ökologische und ökonomische Lebenszyklusanalyse elektrifizierter Antriebsstrangkonzepte. Dissertation, RWTH Aachen, Aachen (2014)

8. German Association of the Automotive Industry: Registration of new vehicles by country 2014 (2015). http://de.statista.com/sta tistik/daten/studie/181566/umfrage/neuzulassungen-von-perso nenkraftwagen-nach-laendern/

9. Hellberg, T.: Nutzungsbasierte Optimierungsstrategie für Antriebssysteme. Dissertation, Helmut-Schmidt-Universität, Hamburg (2016)

10. Hofmann, P.: Hybridfahrzeuge: ein alternatives Antriebssystem für die Zukunft. Springer, Wien [u.a.] (2014)

11. Icha, P.: Evolution of the specific carbon dioxide emission of the german electricity mix from 1990 to 2014 (2015). http://www. umweltbundesamt.de/publikationen/entwicklung-der-spezifischenkohlendioxid-1 (online)

12. Karbowski, D., Haliburton, C., Rousseau, A.: Impact of component size on plug-in hybrid vehicle energy consumption using global optimization (2006). http://www.transportation.anl.gov/ pdfs/HV/460.pdf (online)

13. Meywerk, M.: Vehicle Dynamics. Wiley, Chichester (2015)

14. Mohan, G., Assadian, F., Longo, S.: An optimization framework for comparative analysis of multiple vehicle powertrains. Energies 6(10), 5507-5537 (2013). doi:10.3390/en6105507, http:// www.mdpi.com/1996-1073/6/10/5507

15. Overington, S., Rajakaruna, S.: A modified method for the sizing of the plug-in hybrid electric vehicle propulsion devices. In: Universities Power Engineering Conference (AUPEC), 2011 21st Australasian, pp. 1-7 (2011)

16. Reif, K.: Kraftfahrzeug-Hybridantriebe: Grundlagen, Komponenten, Systeme, Anwendungen, 1st edn. Springer Vieweg, Wiesbaden (2012)

17. SAE J1715: Hybrid electric vehicle (hev) and electric vehicle (ev)terminology (2010)

18. Schulte-Coerne C: Multikriterielle integrierte Systemoptimierung von hybriden Plug-In-Antriebssystemen. Dissertation, RWTH Aachen, Aachen (2015)

19. Sehab, R., Barbedette, B., Chauvin, M.: Electric vehicle drivetrain: Sizing and validation using general and particular mission profiles. In: IEEE International Conference on Mechatronics (ICM), Istanbul, pp. 77-83 (2011)

20. UN/ECE: Uniform provisions concerning the approval of vehicles with regard to the emission of pollutants according to engine fuel requirements. Regulation No. 83 (2015) 